\title{
Spinal intraarterial computed tomography angiography as an effective adjunct for spinal angiography
}

\author{
Shinji Yamamoto, MD, DMSc, Hideaki Kanaya, MD, PhD, and Phyo Kim, MD, PhD, DMSc \\ Department of Neurosurgery, Dokkyo University School of Medicine, Tochigi, Japan
}

OBJECT Spinal digital subtraction angiography (DSA) is indispensable for the precise diagnosis of spinal vascular lesions and the assessment of blood supply to the spinal cord. However, comprehensive spinal DSA covering multiple segments requires repetition of selective catheterization into small segmental arteries, which is time consuming, sometimes difficult, and hazardous. The authors investigated the usefulness of CT angiography with intraarterial contrast injection (IA-CTA) as a preliminary study preceding spinal DSA. With the advent of multidetector CT, it is feasible to obtain images of the spinal cord vasculature instantaneously over a number of segments.

METHODS A total of 56 patients with lesions involving the spinal vasculature underwent IA-CTA with 64- or 320-row detector CT in advance of comprehensive spinal DSA. Contrast material was injected via a pigtail catheter placed at the aorta in proximity to the segments of interest. Scanning was repeated twice to obtain arterial- and venous-phase images to differentiate between the arterial and venous components. The spinal arteries were identified by paging the various multiplanar reconstruction images and tracing the vessels from the aorta. Spinal DSA was subsequently performed by guiding selective catheterization to the feeding segments in reference to the IA-CTA findings. Visualization of the segmental arteries, normal spinal arteries, and abnormal vessels during IA-CTA was investigated and compared with that obtained during spinal DSA.

RESULTS In all 56 patients, spinal IA-CTA successfully enabled visualization of the spinal vessels, including the radicular arteries and the anterior spinal artery. Below the aortic arch, all segmental arteries were identified clearly. The segmental arteries, radiculomedullary arteries, spinal arteries, and abnormal vessels were traced from the aorta, which would be the target of selective catheterization. In $3(6.8 \%)$ patients, IA-CTA revealed severe aortic atherosclerosis and occlusion of some segmental arteries. The information obtained was useful for directing selective catheterization studies. The findings of IA-CTA corresponded well with those of spinal DSA.

CONCLUSIONS IA-CTA is a useful adjunct to spinal DSA for surveying the vasculature surrounding the spinal cord and for orienting selective catheterization. IA-CTA can complement spinal DSA, curtail unnecessary segmental injections, and thus reduce procedural complications.

http://thejns.org/doi/abs/10.3171/2014.12.SPINE14584

KEY WORDS spinal angiography; CT angiography; intraarterial; spinal arteriovenous malformation; spinal arteriovenous fistula; technique

$\mathrm{D}$ IAGNOSIS of spinal vascular disease remains challenging because of the nonspecific clinical symptoms and the limitations of various noninvasive imaging technologies. Precise delineation of vascular anatomy and definition of spinal blood supply are indispensable for optimal treatment.

Spinal digital subtraction angiography (DSA) remains the standard with respect to sensitivity and specificity for diagnosis of spinal vascular lesions., ${ }^{4,6,10,15,16}$ However, comprehensive spinal DSA requires multiple selective catheterization into small segmental arteries and a large volume of contrast material; thus, it carries a risk for complications like vessel dissection and thromboembolism. Major procedure-related complications, such as retroperitoneal bleeding, cerebral ischemia, renal failure, and spinal cord ischemia, have been reported for $1.2 \%-4.6 \%$ of patients. ${ }^{4,6,10,16}$

ABBREVIATIONS CTA = CT angiography; DSA = digital subtraction angiography; IA-CTA = CTA with intraarterial contrast injection; IV-CTA = CTA with intravenous contrast injection.

SUBMITTED June 11, 2014. ACCEPTED December 30, 2014.

INCLUDE WHEN CITING Published online May 29, 2015; DOI: 10.3171/2014.12.SPINE14584.

DISCLOSURE The authors report no conflict of interest concerning the materials or methods used in this study or the findings specified in this paper. None of the authors received financial support from any source for the study here. 
Complete spinal DSA is technically demanding and invasive, particularly in patients with atherosclerosis of the aorta. Effective adjuncts to supplement spinal DSA have been desired.

Enhanced resolution is essential for visualization of minute vessels like spinal arteries. Tomographic images are useful for depicting the precise anatomy of the spinal vessels and adjacent structures. Computed tomography angiography with intraarterial contrast injection (IA-CTA) can provide an extended thin-section scanning range with high-resolution and high-contrast angiographic images. This modality can clearly track the small spinal arteries from the aorta and is useful for identifying the normal spinal arteries (e.g., the artery of Adamkiewicz) safely and reliably for preoperative assessment of thoracoabdominal aortic disease. . $^{14,19}$

Preliminary information regarding the broad and precise vascular anatomy from the aorta to the spinal cord would be useful for making spinal DSA more focused and less invasive. In this study, we performed IA-CTA in advance of comprehensive spinal DSA for visualization of the normal and abnormal spinal arteries, which would be the target of selective catheterization. To assess the effectiveness of spinal IA-CTA, we investigated the detectability of abnormal spinal vessels and normal spinal arteries.

\section{Methods \\ Patients}

This study was approved by the ethical committee of Dokkyo University School of Medicine, and all patients provided written informed consent to participate in the study. The 56 patients in the study had undergone IACTA and spinal DSA for the evaluation of intramedullary arteriovenous malformations $(n=6)$, perimedullary arteriovenous fistulas $(\mathrm{n}=11)$, spinal dural arteriovenous fistulas $(\mathrm{n}=21)$, spinal tumors $(\mathrm{n}=10)$, and miscellaneous spinal cord lesions with suspected vascular pathology that were finally diagnosed as spinal cord infarction or myelitis $(n=8)$ from November 2010 through October 2013. The patient group comprised 36 men and 20 women, 16-84 years of age (mean 51.3 years). Spinal lesions were in the midthoracic to sacral area (below the aortic arch) for 44 patients and in the cervical to upper thoracic area (above the aortic arch) for 12 patients.

\section{Scanning Protocol}

For the first 45 patients, a 64-row multidetector CT unit (SOMATOM sensation, Siemens AG) was used. The scanning parameters comprised $100 \mathrm{kV}, 300 \mathrm{~mA}, 180-\mathrm{mm}$ FOV, 1.0 seconds per rotation, $0.6-\mathrm{mm}$ slice collimation, 1.0 pitch, and $17.3 \mathrm{~mm}$ per second table speed. For the recent 11 patients, a 320-row area-detector CT scanner (Aquilion One, Toshiba Medical Systems Co., Ltd) was used. The parameters were $120 \mathrm{kV}, 8.0$ standard deviation, 180 $\mathrm{mm}$ FOV, 0.5 seconds per rotation, $0.5-\mathrm{mm}$ slice collimation, 1.388 pitch, and $95.7 \mathrm{~mm}$ per second table speed. In an angiography suite, a 4-Fr introducer vessel sheath was inserted into the femoral artery. For contrast injection of IA-CTA, a 4-Fr pigtail catheter was advanced via the vessel sheath under fluoroscopic guidance. To inject the con- trast material in high concentration into the tributary of the spinal cord, we placed the tip of the catheter in the aorta. The positions aimed for were as follows: immediately below the left subclavian artery for middle thoracic to sacral lesions (infra-aortic arch lesions) and immediately above the aortic valve for cervical to upper thoracic lesions (supra-aortic arch lesions). The patients were then transferred to the CT room for IA-CTA. To conduct a broad survey of spinal vessels prior to spinal DSA, we set the scan range of IA-CTA widely: the range was from the upper thorax to the sacrum for infra-aortic arch lesions and from the level of the internal auditory canal to the middle thorax for supra-aortic arch lesions. Scanning was performed in 2 sequences to obtain arterial- and venous-phase images to differentiate between the arterial and venous components. For arterial-phase vascular imaging, a high concentration of contrast material was injected into the arteries. To obtain venous-phase images, scanning was performed as the veins filled following the complete outflow of contrast material from the arteries. The arterial-phase scan began 5 seconds after the injection of iopamidol (300 mg/ $\mathrm{ml}$; Nihon Schering K.K.) through the pigtail catheter at a rate of $3 \mathrm{ml}$ per second. The injection was ceased as the first arterial-phase scanning was completed. After a 15 -second pause, the venous-phase scanning was started. All scanning was performed under the condition of breath holding. After the IA-CTA, patients were returned to the angiography suite. Following the change of angiographic catheter through the femoral vessel sheath, definitive comprehensive spinal DSA was successively performed by the reference of the IA-CTA findings. In this study, to examine the sensitivity of IA-CTA, comprehensive spinal DSA was performed in all segmental arteries encompassing 10 vertebral levels or more without any omission of selective catheterization.

\section{Image Processing and Diagnosis}

The data analysis was performed on a Zio Workstation2 (Ziosoft). Data sets from the arterial- and venous-phase scans were used to reconstruct discrete images of the spinal arteries and veins, which are located close together in the spinal canal. Sagittal, axial, coronal, and oblique coronal multiplanar reconstruction images adjusted to the vertebral curvature were obtained. Reconstruction was done at 2-mm section thicknesses and 1-mm intervals for sagittal, coronal, and oblique coronal multiplanar reconstruction images, and at 2-mm section thicknesses and 5-mm intervals for axial section images. By use of the paging method, spinal arteries were identified from these reconstructed images when continuity between the spinal artery and the aorta could be securely traced. To differentiate between veins and arteries, the temporal sequence was also taken into account; vessels in the first arterial-phase images that were more enhanced in the second venous-phase images were considered venous components. Images of the radicular arteries, normal spinal arteries, and abnormal vessels were studied and compared with DSA images. We evaluated the usefulness of IA-CTA and analyzed the associated scanning time, total volume of contrast material, and complications. 


\section{Results}

For all 56 patients, we successfully obtained images of the spinal vasculature by IA-CTA prior to spinal DSA. For all 44 patients with infra-aortic arch lesions, the segmental and radicular arteries could be visualized clearly within the scanning range. In $3(6.8 \%)$ of these 44 patients, IACTA revealed aortic atherosclerosis and occlusion of some segmental arteries, which was confirmed by spinal DSA. The precise anatomy of arteriovenous malformations and arteriovenous fistulas was depicted at an increased resolution. Information from the bony images facilitated diagnosis of the levels and treatment planning for these lesions. The continuity between the segmental arteries and the aorta could be easily identified by using the paging method. The artery of Adamkiewicz and its origin were identified in all patients with spinal tumors, spinal cord infarction, and myelitis. Among 21 patients with arteriovenous malformations and arteriovenous fistulas at the infra-aortic arch level, the artery of Adamkiewicz and its segmental origin were identified in 7 (33.3\%) patients because of interference from the engorged tortuous vessels. Subsequent complete spinal DSA depicted the normal spinal arteries in $12(57.1 \%)$ of these 21 patients. In patients with supra-aortic arch lesions, all arteriovenous malformations and arteriovenous fistulas could be demonstrated by IA-CTA; however, the resolution of these lesions was relatively low compared with that of infra-aortic lesions due to dilution of the contrast.

Table 1 summarizes the scanning data of IA-CTA and comprehensive DSA. In this protocol of IA-CTA, the scanning time and the total volume of contrast material required were significantly lower when using 320-row detector CT than when using 64-row detector CT. Radiation exposure with 320-row detector CT is higher than that of 64-row detector CT. The protocol of 320-row CTA could be adjusted to reduce unnecessary radiation exposure. There were no complications associated with IA-CTA.

\section{Illustrative Cases \\ Case 1}

A 54-year-old man presented with progressive bilateral lower-extremity paresis and urinary retention (Fig. 1). MRI showed a T2 signal-intensity change from the level of T-6 to the conus medullaris with surrounding flow void signals. Arterial-phase IA-CTA revealed an arteriovenous shunt between the left T-11 intercostal artery and the engorged

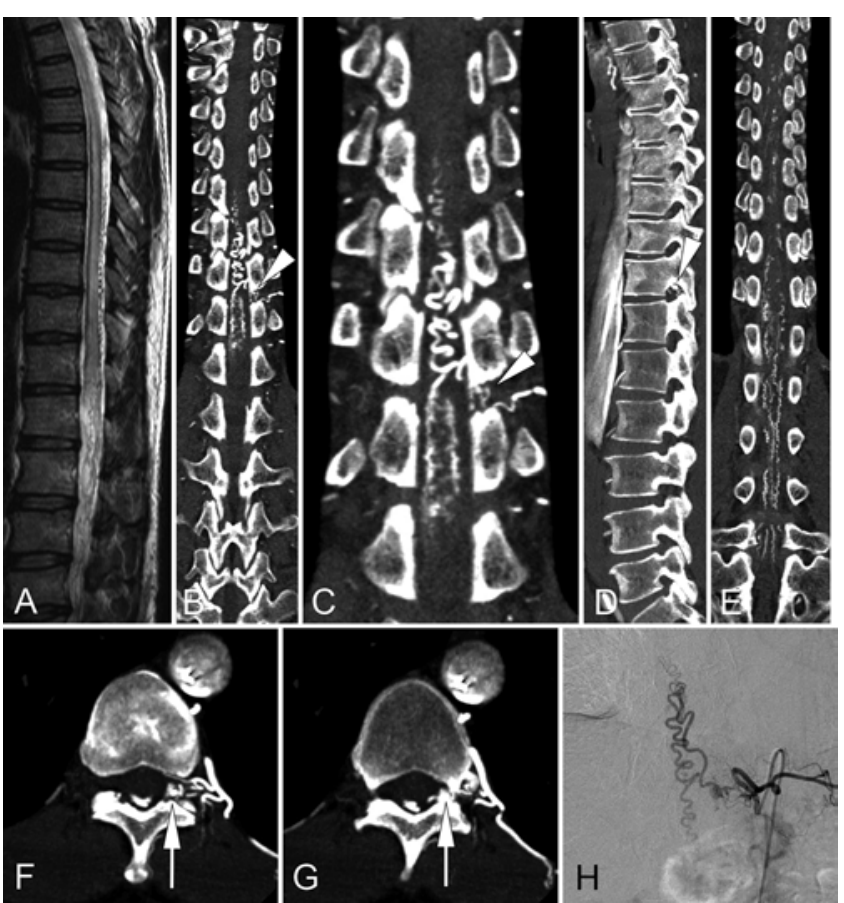

FIG. 1. Case 1. Spinal dural arteriovenous fistula. A: Sagittal T2-weighted MR image showing edema of the spinal cord and perimedullary flow voids. B-G: IA-CTA images showing arteriovenous shunting via the left T-11 intercostal artery (arrowheads) and engorged vascular tangles in the left T11-12 intervertebral foramen and dilated venous plexus (arrows). Arterial-phase coronal sections ( $B$ and $C$ ), an arterial-phase sagittal section (D), venous-phase coronal section (E), and arterial-phase axial sections at the T-11 level ( $F$ and $G)$. $H$ : Selective DSA image of the left T-11 intercostal artery demonstrating equivalent results.

perimedullary veins, connected with a dural arteriovenous fistula. Diffuse reflux of perimedullary blood flow that drained into the bilateral S-1 radicular veins was observed in the venous-phase IA-CTA. The right T-11 intercostal artery was not visualized on IA-CTA. The findings from spinal DSA corresponded with those of IA-CTA, showing occlusion of the right T-11 intercostal artery.

\section{Case 2}

A 38-year-old woman presented with progressively worsening gait disturbance and intermittent low-back pain (Fig. 2). MRI showed intramedullary flow void signals at the T-11 level. IA-CTA demonstrated an intramedullary nidus fed by the left T- 8 and T-12 intercostal arteries. Precise

TABLE 1. Radiation exposure and dye consumption resulting from IA-CTA and comprehensive spinal DSA

\begin{tabular}{|c|c|c|c|c|c|c|c|}
\hline \multirow[b]{2}{*}{ Lesion } & \multirow{2}{*}{$\begin{array}{c}\text { No. } \\
\text { of } \\
\text { Pts }\end{array}$} & \multicolumn{3}{|c|}{ Range (mean) of Values for IA-CTA } & \multicolumn{2}{|c|}{ Range (mean) of Values for DSA } & \multirow[b]{2}{*}{$\begin{array}{l}\text { No. of Segmental } \\
\text { Catheterizations }\end{array}$} \\
\hline & & $\begin{array}{l}\text { Scan Time in } \\
\text { Secs }\end{array}$ & $\begin{array}{l}\text { Contrast Vol } \\
\text { in } \mathrm{ml}\end{array}$ & $\begin{array}{l}\text { Radiation Exposure } \\
\text { in } \mathrm{mGy}\end{array}$ & $\begin{array}{l}\text { Contrast Vol } \\
\text { in } \mathrm{ml}\end{array}$ & $\begin{array}{l}\text { Radiation Exposure } \\
\text { in } \mathrm{mGy}\end{array}$ & \\
\hline Infra-aortic arch lesion* & 36 & $16.9-22.1(20.1)$ & $63-80(72.4)$ & $11.5-28.0(17.8)$ & $102-150(120.0)$ & $1065-2013(1468.2)$ & $20-26(22.4)$ \\
\hline Supra-aortic arch lesion* & 9 & $10.1-11.0(10.7)$ & $45-50(47.1)$ & $12.2-24.5(16.9)$ & $80-95(80.5)$ & $688-1158(879.5)$ & $10-12(10.2)$ \\
\hline Infra-aortic arch lesiont & 8 & $4.01-4.47(4.34)$ & $27-30(27.9)$ & $20.9-31.6(26.8)$ & $105-140(124.6)$ & $1042-2066(1624.9)$ & $20-26(20.5)$ \\
\hline Supra-aortic arch lesion† & 3 & $2.38-2.93(2.71)$ & $24-30(25.1)$ & $12.5-20.6(18.0)$ & 85-108 (89.3) & $714-956(857.0)$ & $10-12(10.3)$ \\
\hline
\end{tabular}




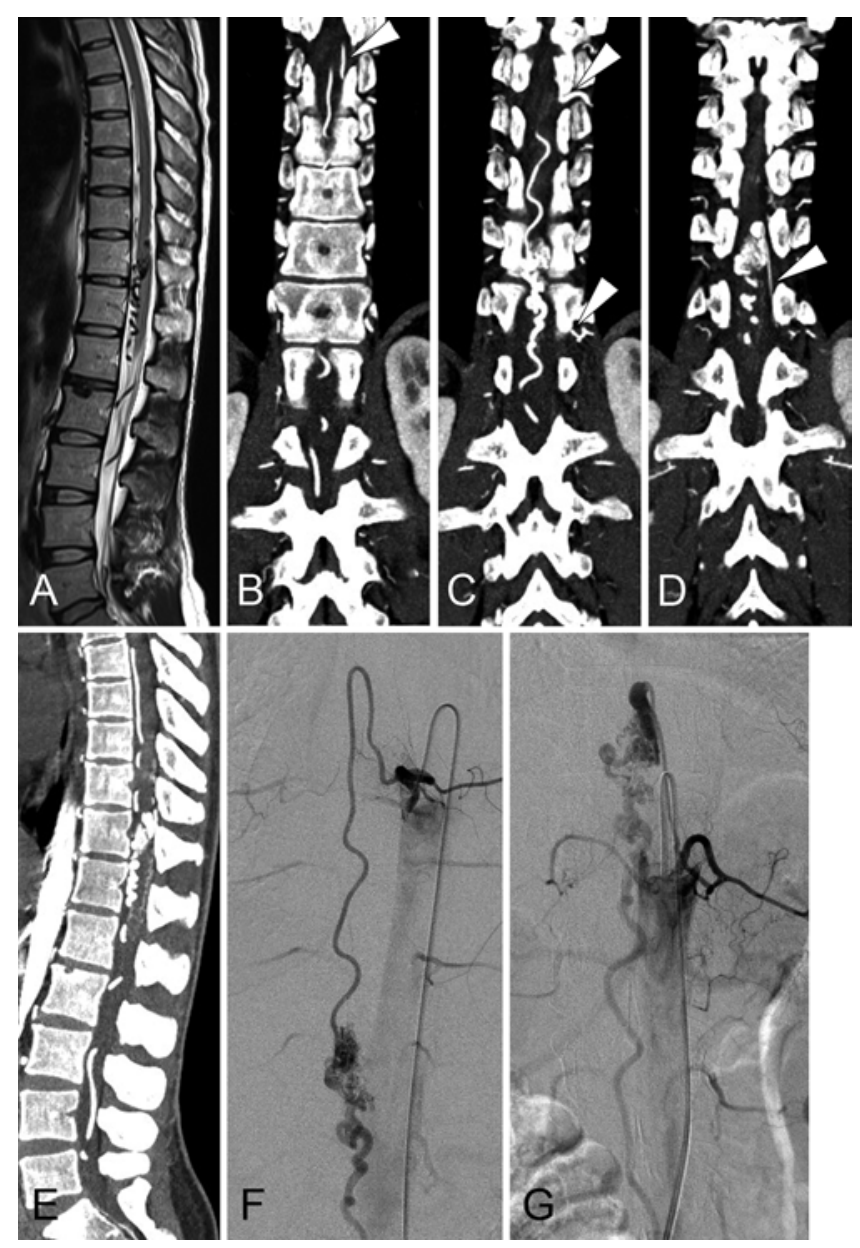

FIG. 2. Case 2. Spinal cord arteriovenous malformation. A: Sagittal T2-weighted MR image showing perimedullary and intramedullary flow void signals. B-D: Arterial-phase coronal images of IA-CTA demonstrating the lesion fed by the left T-8 and T-12 intercostal arteries (arrowheads). E: Sagittal arterial-phase IA-CTA image showing intramedullary varix. $F$ and $G$ : Selective DSA images demonstrating the identical findings at left T-8 (F) and left T-12 (G).

location of the intramedullary nidus and the varix were visualized. Spinal DSA substantiated the IA-CTA findings.

\section{Case 3}

A 48-year-old man presented with gradually progressing dysesthesia and weakness of bilateral lower extremities and urinary retention (Fig. 3). MRI demonstrated intramedullary high-intensity signal from the T-8 level to the conus medullaris with perimedullary flow void signals on T2-weighted images. IA-CTA clearly showed an arteriovenous shunt between the left T-11 intercostal artery and the engorged perimedullary veins. Some branched vascular tangles were present along the root sleeve, comprising arteriovenous shunting at the left T11-12 intervertebral foramen. The reconstructed images indicated no other vascular abnormalities. Spinal DSA depicted a spinal dural arteriovenous fistula at the left T11-12 intervertebral foramen. The refluxed anterior internal vertebral plexus was isolated from the other venous system, which corresponded with the findings of IA-CTA.

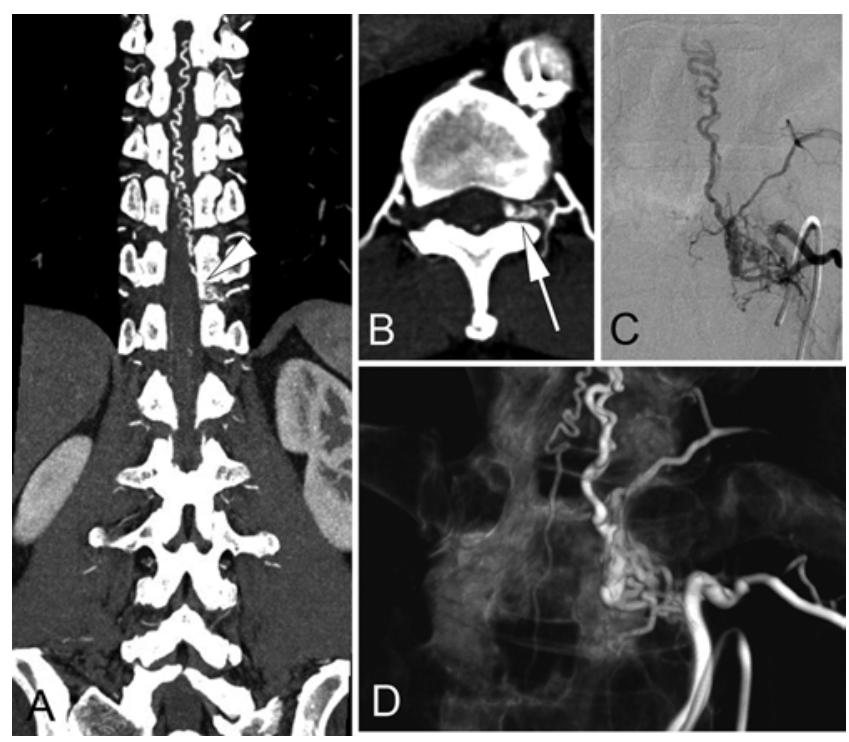

FIG. 3. Case 3. Spinal dural arteriovenous fistula. A: Arterial-phase coronal IA-CTA image showing arteriovenous shunting arising from the left T-11 intercostal artery through the isolated venous plexus (arrowhead). B: Arterial-phase axial IA-CTA image at the T-11 level demonstrating branched vascular tangles along the root sleeve (arrow). $\mathrm{C}$ and D: Selective DSA images (2D [C] and 3D [D]) of the left T-11 intercostal artery substantiating the IA-CTA findings.

\section{Case 4}

A 48-year-old man presented with urinary hesitancy and weakness of bilateral lower extremities (Fig. 4). MRI showed spinal cord swelling from the T-7 level to the conus medullaris, with surrounding perimedullary flow voids. Intramedullary flow void signals were observed at the T-7 level. CTA with intravenous contrast injection (IV-CTA) performed at a local hospital demonstrated abnormal vasculature of the spinal cord, but the precise anatomy of the lesion was obscure. IA-CTA demonstrated engorged perimedullary vessels fed from the right T-9 and left T-8 intercostal arteries. The origin of the artery of Adamkiewicz was suspected to be at the left T-11 intercostal artery; however, the finding was uncertain because of the adjacent dilated abnormal vessels. Spinal DSA disclosed the spinal cord arteriovenous fistula fed by the above-mentioned arteries and identified the origin of the artery of Adamkiewicz at the left T-11 intercostal artery.

\section{Case 5}

A 59-year-old man presented with progressing paraparesis, urinary disturbance, and constipation (Fig. 5). At a local hospital, embolization of a filum terminale arteriovenous fistula had been performed via the left lateral sacral artery and the left T-12 intercostal artery; however, the patient's symptoms continued to be progressive. Postoperative MRI, MR angiography, and IV-CTA images taken at the local hospital demonstrated persistent cord swelling and perimedullary abnormal vessels, which indicated the remnant of the arteriovenous shunt. Severe aortic atherosclerosis and tortuosity limited selective catheterization into the segmental arteries, and incomplete DSA failed to enable visualization of the fistula. IA-CTA revealed the 

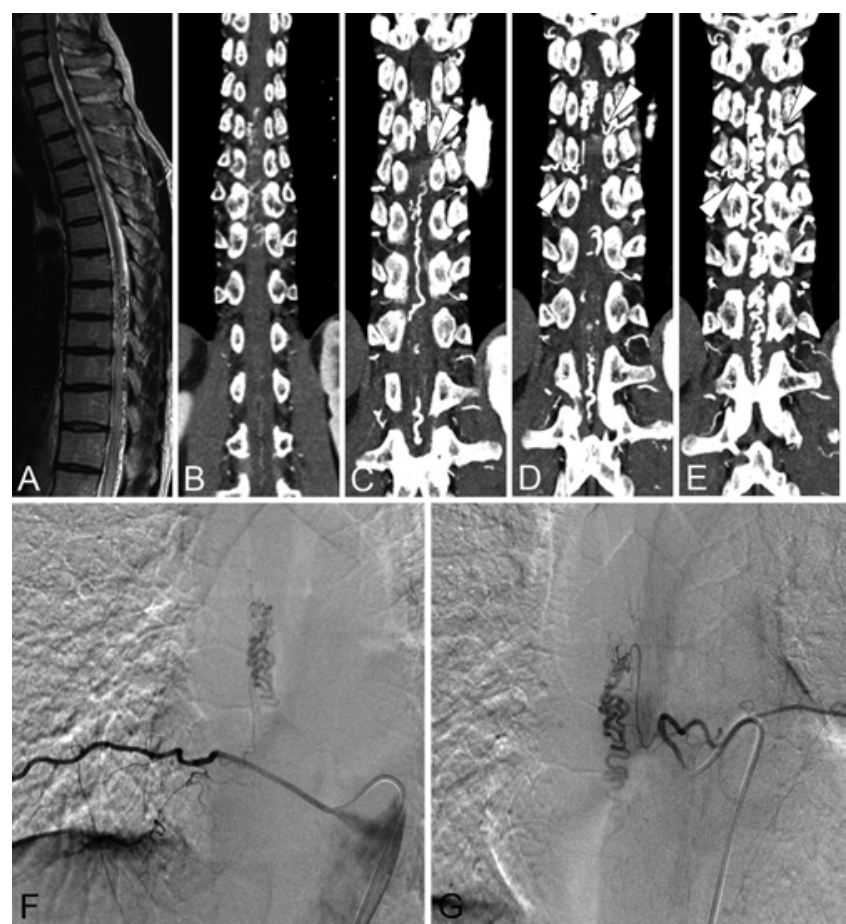

FIG. 4. Case 4. Spinal cord arteriovenous fistula. A: Sagittal T2-weighted MR image showing spinal cord swelling and perimedullary flow void signals. B: Multiplanar reconstruction coronal image of IV-CTA indicating abnormal spinal vessels but not identifying the feeders. C-E: Coronal arterial-phase IA-CTA images demonstrating the lesion fed by the right T-9 and left T-8 intercostal arteries (arrowheads). F and G: Selective DSA images demonstrating the same findings at right $T-9(F)$ and left T-8 $(G)$. remnant of arteriovenous fistula and dilated anterior spinal artery, which was supplied by the left T-6 intercostal artery. Extensive atherosclerosis and the resultant occlusion of the orifices of the right T-9, right T-11, and left T-10 intercostal arteries as well as severe aortic tortuosity were evident. On a repeated session of spinal DSA, selective catheterization into the left T-6 intercostal artery was not feasible because of the severe aortic tortuosity. Microsurgical obliteration of the remnant arteriovenous fistula was planned.

\section{Case 6}

A 40-year-old man presented with sudden onset of nuchal pain and dysesthesia of the right upper extremity (Fig. 6). CT and MRI revealed a dense subarachnoid hemorrhage in the cervical spine and the posterior fossa. IACTA showed perimedullary arteriovenous shunting at the ventral surface of the C-5 level of the spinal cord, which was fed by the bilateral C-5 radicular arteries and drained into the right vertebral venous plexus. DSA showed a perimedullary arteriovenous fistula (Type I) fed by the bilateral C-5 radicular arteries arising from the vertebral arteries and draining via the right $\mathrm{C}-5$ radicular vein. Subsequent DSA revealed identical pathology.

\section{Discussion}

By using IA-CTA, we successfully visualized a wide range of spinal arteries, which could be tracked to the aorta and distinguished from the spinal veins. The results provide useful information for orienting selective catheterization to the targets in advance of spinal DSA. IACTA could also demonstrate pathological changes of the aorta in conjunction with the segmental arteries. Dilation and tortuosity of the aorta may preclude selective catheter-
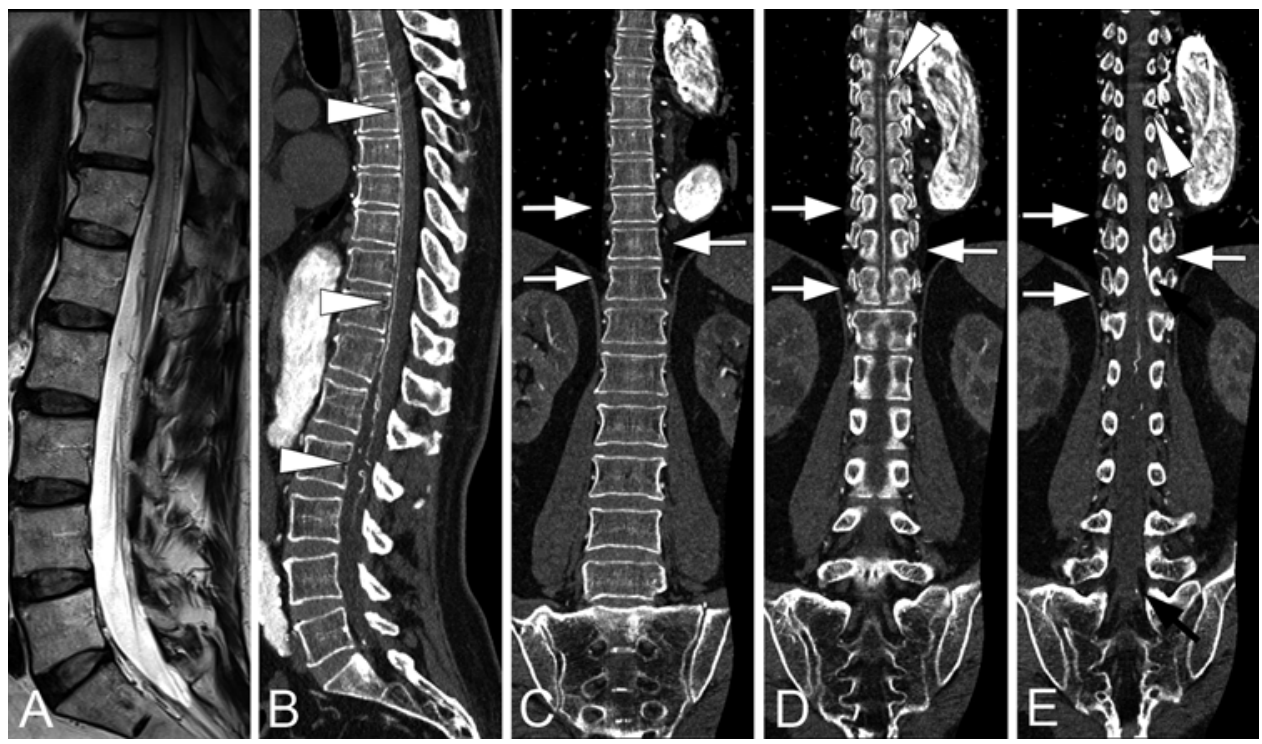

FIG. 5. Case 5. Filum terminale arteriovenous fistula. A: Sagittal T2-weighted MR image showing persistent cord edema and perimedullary flow voids. B: Sagittal arterial-phase IA-CTA image demonstrating dilated anterior spinal artery continuing to the arteriovenous fistula at the filum terminale (arrowheads). C-E: Multiplanar reconstruction coronal images of IA-CTA (arterial phase) demonstrating the lesion fed by the left T-6 intercostal artery (arrowheads) through the dilated anterior spinal artery and the obliteration of the intercostal arteries of right T-9, left T-10, and right T-11 (arrows). Black arrows indicate 2 previously embolized feeders. 


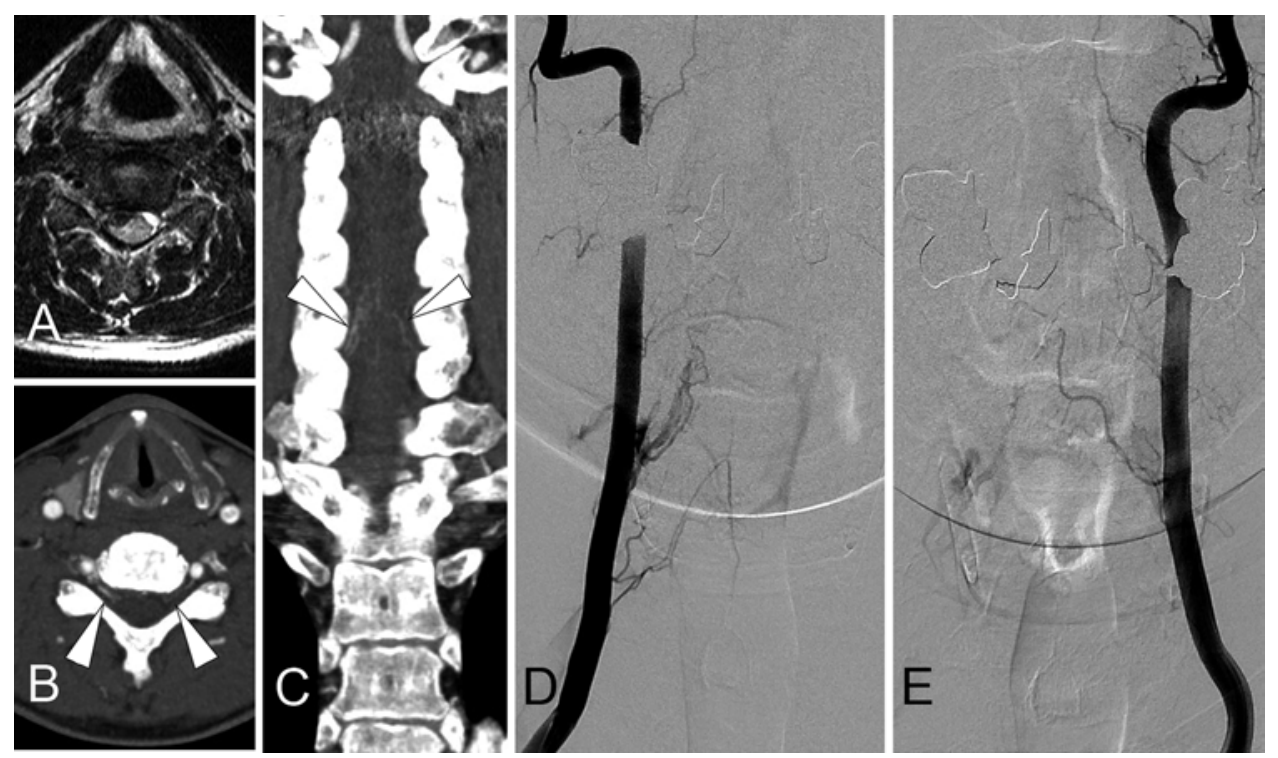

FIG. 6. Case 6. Spinal cord arteriovenous fistula. A: Axial T2-weighted MR image showing a subarachnoid hematoma on the ventral surface of the spinal cord at the C-5 level. B and C: Arterial-phase IA-CTA images (axial section at C-5 level [B] and coronal [C]) indicating arteriovenous shunting fed by the bilateral C-6 radicular arteries located at the ventral surface of the cord (arrowheads). D and E: Bilateral vertebral DSA images demonstrating a spinal cord arteriovenous fistula fed by the right and left C-6 radicular arteries (right [D] and left [E] vertebral artery injections).

ization into the segmental arteries. Aortic atherosclerosis may also obliterate the orifices of the intercostal and/or lumbar arteries..$^{15} \mathrm{We}$ could detect these alterations prior to conducting spinal DSA, which was useful for reducing the risks of causing vascular injury and thromboembolism pertinent to the selective wedging. To the best of our knowledge, this is the first report describing the effectiveness of IA-CTA as a survey to guide selective catheterization for spinal vascular lesions.

Noninvasive imaging technologies for the detection of spinal vascular lesions have been developed. Multiphase contrast-enhanced MR angiography can enable visualization of large normal spinal vessels and pathological vascular lesions but still requires a long acquisition time with limited scanning range and low spatial resolution., ${ }^{2,8,9,21,24}$ Multidetector CT requires only a very short acquisition time and provides a wider scanning range and high spatial and temporal resolution. As a result, small spinal blood vessels that extend craniocaudally can be effectively visualized. The tomographic nature of CTA is very useful for analyzing the precise anatomy of the spinal vessels and adjacent structures. ${ }^{3,5,7,11,12,17,18,20,22,23,25}$ The radicular and spinal arteries are small vessels with diameters of $0.5-1.5$ $\mathrm{mm}$ that run very close to the osseous structures. The anatomical features reduce the contrast-to-noise ratio, and the radicular arteries and their continuities with the aorta are usually obscured in IV-CTA. MR angiography and IVCTA therefore provide limited information for assessment of the precise anatomy of complex spinal vascular lesions.

To delineate spinal vessels clearly, a method that enhances the bolus characteristic of contrast administration is preferable. Sharp rise and washout of the focal distribution of the contrast enhances the spatial and temporal resolutions. Intravenously administered contrast medium is mixed in the right heart, diluted as it passes through the pulmonary circulation, and partially flows into the spinal vessels. It has been reported that intravenous injection of contrast medium at a rate exceeding $4 \mathrm{ml}$ per second did not significantly enhance visualization of arteries. ${ }^{13} \mathrm{In}$ this regard, intraarterial injection is effective for inducing a sharp rise of the concentration of the contrast medium. With IA-CTA, it is possible to inject a large amount of contrast medium at a high concentration in a short time directly into the tributary so that rapid distribution is effectively achieved, which is not possible with intravenous contrast injection.

For adequate treatment of the pathology and prevention of compromised spinal cord blood flow during surgery and endovascular treatment, a complete angiographic study is needed to delineate the abnormality and define the anatomical features of the blood supply to the spinal cord in the vicinity of the lesion. Spinal DSA with selective catheterization has the highest temporal and spatial resolution and can provide the tomographic and volumetric reconstructive images with the advent of 3D C-arm cone-beam CTA. ${ }^{1}$ Selective DSA is the gold standard for the definitive diagnosis of spinal vascular lesions. However, it is difficult to visualize multiple spinal segmental arteries simultaneously with DSA. Remote segmental arteries may feed the spinal lesion via longitudinally extending perimedullary spinal arteries. Thus, multiple catheterizations into the segmental arteries are indispensable for a complete study, especially with respect to the thoracolumbar lesions. IACTA can delineate a broad range of spinal arteries arising from multiple segmental arteries. With the image of adjacent bony structures, high-resolution angiographic images help us to identify the precise anatomy of the lesions, including the levels of the feeding arteries. Multiphase dynamic imaging, facilitated by the sharp rise and fall of the contrast distribution, can discriminate the arterial 
component from the draining veins, which improves the precision of the diagnosis. This information can orient the surgeon and focus catheter placement and lead to reduction in the number of selective catheterizations and fewer procedural complications. In this series, to examine the effectiveness of IA-CTA, especially regarding its sensitivity, comprehensive spinal DSA was performed in all segmental arteries encompassing 10 or more vertebral levels without any omission of selective catheterization. Thus, multiple selective catheterization and injection resulted in a large volume of dye consumption and radiation exposure in spinal DSA. Our results demonstrate that the selective angiograms for many segmental arteries separated from the targets could be omitted by the reference of IA-CTA findings. When the target vessels are confirmed and no remote abnormal vessel is detected by IA-CTA, we can focus on several vertebral levels around the targets considering the presence of epidural/paraspinal anastomoses. Even in diffuse large lesions, IA-CTA can delineate the negative findings remote from the lesion, which is also useful for curtailing unnecessary selective catheterization.

In this study, we obtained the radiation dose output from each procedure. Radiation exposure by the spinal IA-CTA was confined to $16.9-26.8 \mathrm{mGy}$, which corresponds to that of standard contrast-injection abdominal CT scanning. On the other hand, complete spinal DSA over multiple segments required high radiation exposure: $857.0-879.5 \mathrm{mGy}$ for supra-aortic arch lesions and 1468.2-1624.9 mGy for infra-aortic arch lesions. It is not possible to directly compare the radiation exposure of IA-CTA with that of DSA; however, omission of unnecessary selective DSA by the reference of IA-CTA can significantly reduce radiation exposure. IA-CTA is effective not only as a broad survey, but for reduction of dye consumption and radiation exposure.

The most common clinical indication for spinal DSA is a suspicion of arteriovenous malformations or arteriovenous fistulas. Other spinal lesions like spinal tumors and miscellaneous spinal cord lesions with possible vascular abnormalities can be examined by spinal DSA. In this study, 10 large spinal tumors involving normal vessels and/or accompanying vascular abnormalities, such as large intramedullary hemangioblastomas with engorged serpentine flow void signals and huge spinal cervical sarcomas encasing bilateral vertebral arteries, were evaluated preoperatively to detect pathological arteriovenous shunts and to determine the precise location of normal spinal arteries and abnormal tumor feeders. Noninvasive modalities like MR angiography and conventional IV-CTA cannot provide the information precisely. For preoperative angiographic evaluation of the tumor, IA-CTA can determine the target vessels in advance of spinal selective DSA. The diagnosis of spinal vascular disease is sometimes difficult because of its nonspecific clinical symptoms and limitations of the various noninvasive imaging technologies. Misdiagnosis or delayed diagnosis can lead to worsening myelopathy and diminished prognosis for recovery. The sensitivity of MRI for detecting abnormal spinal vasculature is not high. Chen et al. reported that MRI findings showed 51\% sensitivity and $83 \%$ specificity for spinal vascular malformations. ${ }^{4}$ They also reported that, among 45 patients who had previously received a diagnosis of and treatment for transverse myelitis, spinal DSA revealed a spinal vascular malformation in $14(31 \%)$ patients. Although the data were affected by patient selection bias, these results suggest that angiographic examination of patients with atypical presentations or poor responses to therapy may reveal spinal vascular pathology. In our study, 10 miscellaneous spinal cord lesions with atypical clinical presentations were evaluated precisely by IA-CTA and spinal DSA for possible vascular lesions. In 2 patients, spinal dural arteriovenous fistulas were found, and for the remaining 8 patients, spinal cord infarction or myelitis of unknown etiology were diagnosed and managed. For diagnosis of possible spinal vascular lesion undetermined by various noninvasive imaging techniques, IA-CTA is useful for indicating the target vessels in advance of definitive spinal DSA.

Spinal IA-CTA has some limitations. Its effectiveness for cervical to upper thoracic lesions (supra-aortic arch lesions) is limited because of the anatomical features of the arterial supply. To inject the contrast material into the tributary at a higher concentration, the catheter tip has to be placed at a suitable position. For cervical and upper thoracic lesions, the tip must be advanced at the proximal portion of the ascending aorta because the bilateral vertebral arteries, external carotid arteries, deep cervical arteries, ascending cervical arteries, and supreme intercostal arteries constitute the potential source of blood supply. The major outflow of contrast material into the descending aorta and upper limbs causes insufficiently robust contrast filling at the lesion. Selective catheterizations for these arteries are not difficult compared with those for infra-aortic arch lesions. To detect tiny normal spinal arteries in the presence of a concomitant large vascular malformation, IA-CTA is inadequate. DSA with selective catheterization has the highest temporal and spatial resolution, and detailed hemodynamic and anatomical analysis of spinal vessels can currently be provided only by selective DSA. The effectiveness of IA-CTA as a substitute for spinal DSA is to be established in the future with further improvement of CT resolution. Image quality of IA-CTA is susceptible to the scanning range, table speed of CT equipment, injection speed of the contrast material, and blood flow velocity. In high-flow arteriovenous shunting lesions, both the arterial and venous components can be depicted in the first arterial-phase IA-CTA. On the other hand, in patients with systemic delayed blood flow such as in severe cardiac failure, the arterial component may be visualized in the venous-phase images despite the 15-second pause between the arterial and venous phases. For optimal visualization, the scanning protocol should be adjusted to the condition. Placement of the catheter into the aorta renders IA-CTA more invasive than IV-CTA or MR angiography. However, the combination of IA-CTA and spinal DSA is safer and less time consuming than stand-alone spinal DSA. We have not yet experienced any complications related to IA-CTA preceding spinal DSA. The total dose of contrast material used in the studies combined was comparable with that used in spinal DSA alone.

\section{Conclusions}

IA-CTA is a useful adjunct to spinal DSA for surveying the vasculature surrounding the spinal cord and for orient- 
ing selective catheterization. The combination of IA-CTA and spinal DSA is safe and effective, improves diagnostic accuracy, and reduces complications.

\section{References}

1. Aadland TD, Thielen KR, Kaufmann TJ, Morris JM, Lanzino G, Kallmes DF, et al: 3D C-arm conebeam CT angiography as an adjunct in the precise anatomic characterization of spinal dural arteriovenous fistulas. AJNR Am J Neuroradiol 31:476-480, 2010

2. Bley TA, Duffek CC, François CJ, Schiebler ML, Acher CW, Mell M, et al: Presurgical localization of the artery of Adamkiewicz with time-resolved 3.0-T MR angiography. Radiology 255:873-881, 2010

3. Boll DT, Bulow H, Blackham KA, Aschoff AJ, Schmitz BL: MDCT angiography of the spinal vasculature and the artery of Adamkiewicz. AJR Am J Roentgenol 187:1054-1060, 2006

4. Chen J, Gailloud P: Safety of spinal angiography: complication rate analysis in 302 diagnostic angiograms. Neurology 77:1235-1240, 2011

5. Cognard C, Miaux Y, Pierot L, Weill A, Martin N, Chiras J: The role of CT in evaluation of the effectiveness of embolisation of spinal dural arteriovenous fistulae with N-butyl cyanoacrylate. Neuroradiology 38:603-608, 1996

6. Forbes G, Nichols DA, Jack CR Jr, Ilstrup DM, Kispert DB, Piepgras DG, et al: Complications of spinal cord arteriography: prospective assessment of risk for diagnostic procedures. Radiology 169:479-484, 1988

7. Guillevin R, Vallee JN, Cormier E, Lo D, Dormont D, Chiras J: N-butyl 2-cyanoacrylate embolization of spinal dural arteriovenous fistulae: CT evaluation, technical features, and outcome prognosis in 26 cases. AJNR Am J Neuroradiol 26:929-935, 2005

8. Hyodoh H, Kawaharada N, Akiba H, Tamakawa M, Hyodoh K, Fukada J, et al: Usefulness of preoperative detection of artery of Adamkiewicz with dynamic contrast-enhanced MR angiography. Radiology 236:1004-1009, 2005

9. Kawaharada N, Morishita K, Hyodoh H, Fujisawa Y, Fukada J, Hachiro Y, et al: Magnetic resonance angiographic localization of the artery of Adamkiewicz for spinal cord blood supply. Ann Thorac Surg 78:846-852, 2004

10. Kieffer E, Fukui S, Chiras J, Koskas F, Bahnini A, Cormier E: Spinal cord arteriography: a safe adjunct before descending thoracic or thoracoabdominal aortic aneurysmectomy. J Vasc Surg 35:262-268, 2002

11. Kudo K, Terae S, Asano T, Oka M, Kaneko K, Ushikoshi $\mathrm{S}$, et al: Anterior spinal artery and artery of Adamkiewicz detected by using multi-detector row CT. AJNR Am J Neuroradiol 24:13-17, 2003

12. Lai PH, Weng MJ, Lee KW, Pan HB: Multidetector CT angiography in diagnosing type I and type IVA spinal vascular malformations. AJNR Am J Neuroradiol 27:813-817, 2006

13. Nakayama Y, Awai K, Yanaga Y, Nakaura T, Funama Y, Hirai T, et al: Optimal contrast medium injection protocols for the depiction of the Adamkiewicz artery using 64-detector CT angiography. Clin Radiol 63:880-887, 2008

14. Nojiri J, Matsumoto K, Kato A, Miho T, Furukawa K, Ohtsubo $\mathrm{S}$, et al: The Adamkiewicz artery: demonstration by intra-arterial computed tomographic angiography. Eur J Cardiothorac Surg 31:249-255, 2007
15. Oldfield EH, Bennett A III, Chen MY, Doppman JL: Successful management of spinal dural arteriovenous fistulas undetected by arteriography. Report of three cases. J Neurosurg 96 (2 Suppl):220-229, 2002

16. Savader SJ, Williams GM, Trerotola SO, Perler BA, Wang MC, Venbrux AC, et al: Preoperative spinal artery localization and its relationship to postoperative neurologic complications. Radiology 189:165-171, 1993

17. Si-jia G, Meng-wei Z, Xi-ping L, Yu-shen Z, Jing-hong L, Zhong-hui W, et al: The clinical application studies of CT spinal angiography with 64-detector row spiral CT in diagnosing spinal vascular malformations. Eur J Radiol 71:2228,2009

18. Takase K, Sawamura Y, Igarashi K, Chiba Y, Haga K, Saito $\mathrm{H}$, et al: Demonstration of the artery of Adamkiewicz at multi-detector row helical CT. Radiology 223:39-45, 2002

19. Uotani K, Yamada N, Kono AK, Taniguchi T, Sugimoto K, Fujii M, et al: Preoperative visualization of the artery of Adamkiewicz by intra-arterial CT angiography. AJNR Am J Neuroradiol 29:314-318, 2008

20. Utsunomiya D, Yamashita Y, Okumura S, Urata J: Demonstration of the Adamkiewicz artery in patients with descending or thoracoabdominal aortic aneurysm: optimization of contrast-medium application for 64-detector-row CT angiography. Eur Radiol 18:2684-2690, 2008

21. Yamada N, Takamiya M, Kuribayashi S, Okita Y, Minatoya K, Tanaka R: MRA of the Adamkiewicz artery: a preoperative study for thoracic aortic aneurysm. J Comput Assist Tomogr 24:362-368, 2000

22. Yamaguchi S, Eguchi K, Kiura Y, Takeda M, Nagayama T, Uchida H, et al: Multi-detector-row CT angiography as a preoperative evaluation for spinal arteriovenous fistulae. Neurosurg Rev 30:321-327, 2007

23. Yamaguchi S, Nagayama T, Eguchi K, Takeda M, Arita K, Kurisu K: Accuracy and pitfalls of multidetector-row computed tomography in detecting spinal dural arteriovenous fistulas. J Neurosurg Spine 12:243-248, 2010

24. Yoshioka K, Niinuma H, Ehara S, Nakajima T, Nakamura M, Kawazoe K: MR angiography and CT angiography of the artery of Adamkiewicz: state of the art. Radiographics 26 (Suppl 1):S63-S73, 2006

25. Zampakis P, Santosh C, Taylor W, Teasdale E: The role of non-invasive computed tomography in patients with suspected dural fistulas with spinal drainage. Neurosurgery 58:686-694, 2006

\section{Author Contributions}

Conception and design: Yamamoto, Kim. Acquisition of data: Yamamoto, Kanaya. Analysis and interpretation of data: all authors. Drafting the article: Yamamoto, Kim. Critically revising the article: Yamamoto, Kim. Reviewed submitted version of manuscript: all authors. Approved the final version of the manuscript on behalf of all authors: Yamamoto. Statistical analysis: Yamamoto. Administrative/technical/material support: Yamamoto, Kanaya. Study supervision: Kim.

\section{Correspondence}

Shinji Yamamoto, Department of Neurosurgery, Dokkyo University School of Medicine, 880 Kitakobayashi, Mibu, Tochigi 3210293, Japan. email: shinjiyamamoto2-nsu@umin.ac.jp. 